\title{
Implementation of a Formal Debriefing Program After Pediatric Rapid Response Team Activations
}

\author{
Linda Aponte-Patel, MD \\ Arash Salavitabar, MD \\ Pamela Fazzio, MD
}

\author{
Andrew S. Geneslaw, MD \\ Pamela Good, MD \\ Anita I. Sen, MD
}

\section{ABSTRACT}

Background Debriefing after pediatric rapid response team activations (RRT-As) in a tertiary care children's hospital was identified to occur only sporadically. The lack of routine debriefing after RRT-As was identified as a missed learning opportunity.

Objective We implemented a formal debriefing program and assessed staff attitudes toward and experiences with debriefing after pediatric RRT-As.

Methods Real-time feedback for pediatrics residents captured clinical and debriefing data for each RRT-A from July 2014 to June 2016. The debriefing on physiology, team communication, and anticipation of clinical deterioration was introduced in July 2015. To assess debriefing perceptions, residents, intensive care fellows, nurses, and respiratory therapists participated in anonymous preintervention and postintervention surveys. We also developed a workshop to teach residents how to lead debriefing.

Results Debriefing after RRT-As increased from $26 \%$ preintervention to $46 \%$ postintervention $(P<.0001)$. A total of 43 of 76 pediatrics residents (57\%) attended at least 1 of 4 debriefing workshops. Both preintervention and postintervention, more than $80 \%$ (70 of 78 preintervention and 54 of 65 postintervention) of health professionals surveyed strongly agreed or agreed that there was a benefit to debriefing after RRT-As. Postintervention, 65\% (26 of 40) of respondents strongly agreed or agreed that debriefing improved their understanding of the RRT-A process. The rate of debriefing was sustained at $46 \%$ ( 6 months after the end of the study period).

Conclusions Debriefing frequency after pediatric RRT-As significantly increased with the introduction of a formal debriefing program. A majority of health professionals and trainees reported this practice was a valuable experience.

\section{Introduction}

Rapid response teams (RRTs) have become the standard of care over the last decade, as research has shown them to be associated with reduced mortality and rate of cardiopulmonary arrests outside the intensive care unit. ${ }^{1,2}$ Postarrest debriefing has become increasingly common following the 2010 American Heart Association resuscitation guidelines to include this practice to improve future clinical performance. ${ }^{3}$ Debriefing requires learners to organize their thoughts after clinical scenarios, ${ }^{4}$ and has been shown to teach teamwork and communication. ${ }^{5}$ Debriefing is also an important feature of simulationbased teaching. ${ }^{6-8}$

Simulation and arrest literature support using debriefing for education, but no literature specifically related to debriefing after RRT activations (RRT-As) exists. The lack of routine debriefing after RRT-As is a missed learning opportunity. The purpose of this study was to determine the feasibility and sustainability of implementing a formal, resident-led, debriefing program after RRT-As.

DOI: http://dx.doi.org/10.4300/JGME-D-17-00511.1

\section{Methods}

This study took place at an academic, urban, tertiary care children's hospital with approximately 200 pediatric beds, including 41 combined pediatric intensive care unit (PICU) and cardiac intensive care unit beds. Our pediatric RRT consists of a PICU nurse and a respiratory therapist. The RRT responds to calls from the pediatric floors and other select locations, including dialysis, magnetic resonance imaging, and outpatient oncology. Pediatrics residents and PICU fellows receive page notifications of RRTAs, but they are not required to attend. Pediatrics residents attend most RRT-As, and PICU fellows attend at the request of the PICU nurse if there is a clinical indication for their immediate assessment. RRT-A data, including frequency, location, demographics, and outcomes, are collected by the hospital's arrest committee chair and are discussed monthly.

A real-time RRT-A feedback form and preintervention and postintervention surveys were used to collect data. The real-time RRT-A feedback form was designed to assess crowd control, resistance to activating the RRT, timing of admission, PICU fellow attendance, and participation in debriefing. This form, consisting of 10 questions, was completed privately by residents for each RRT-A within a few 
days of the event. Data regarding debriefing practices using the real-time RRT-A feedback form were collected preintervention (July 2014 to June 2015) and postintervention (July 2015 to June 2016).

The second tool, an online survey, was designed by the authors, without further testing, to assess aspects of debriefing after RRT-As, including the perceived frequency of debriefing and interest in debriefing after RRT-As; the ideal duration, composition, and timing of debriefing; and changes in RRT-A participants' opinions about debriefing. The survey consisted of 13 preintervention (February 2015) and 17 postintervention (May 2016) questions using Likert scales, forced-choice format, and a single free-text response. An invitation to complete the voluntary, anonymous survey using SurveyMonkey was e-mailed to RRT-A participants (pediatrics residents, PICU nurses, PICU fellows, and respiratory therapists). After completion, respondents were invited to enter a raffle for a \$20 gift card. We used the results of the preintervention survey to develop and introduce a formal debriefing program for RRT-As in July 2015.

Our primary intervention consisted of the introduction of pediatrics resident-led debriefing huddles after RRT-As, with goals that debriefing should occur by the end of the RRT's 12-hour shift, last 2 to 10 minutes, and include the interdisciplinary team that participated in the event. Three topics were suggested for debriefing based on themes identified from the first survey and debriefing best practices ${ }^{9}$ : (1) shared understanding of physiology; (2) team communication; and (3) anticipation and/or avoidance of the given RRT event. Leaders of the debriefing were encouraged to summarize the discussion with a teaching point.

To provide directed learning, ${ }^{10}$ a 1 -hour workshop was designed to teach pediatrics residents debriefing techniques. The workshop began with a reflective exercise with the following prompts: "Describe a memorable RRT-A." "Why was it memorable?" "What did you learn?" "Did your team debrief?" and "How did debriefing or not debriefing affect your learning?" A review of preintervention survey results and institution-specific RRT-A data were presented. Literature demonstrating successful debriefing programs at children's hospitals ${ }^{11}$ and novices successfully leading debriefing ${ }^{12}$ was reviewed. Finally, a novel acronym to standardize debriefing after RRT-As was introduced, named DEBRIEF (Day of, Everybody is invited, Be brief, Review the case, Improve team communication, Earlier intervention [could an event be anticipated], Find a learning point). The workshop ended with role-play exercises to allow for practice using the acronym.
What was known and gap

Infrequent debriefings after pediatric rapid response team activations may be a missed learning opportunity.

\section{What is new}

Real-time feedback after rapid response team activations, focusing on physiology, team communication, and anticipating clinical deterioration, and a workshop to teach residents how to lead debriefing.

\section{Limitations}

Single specialty, single institution study, and low response rate limit generalizability.

\section{Bottom line}

Debriefing frequency increased after the formal debriefing program, and it was reported to be a valuable experience for health professionals and trainees.

Run charts were posted monthly on each pediatric floor displaying the number of RRT-As, the frequency of RRT-As within 6 hours of admission, the frequency of RRT-As leading to PICU admission, and the frequency of RRT-As with debriefing. Chief residents included reminders to pediatrics residents about debriefing in periodic e-mails, and during orientation at the beginning of each clinical rotation. Additionally, in the postintervention period, RRT morning report resident conferences highlighted issues surrounding specific RRT-As, and increased awareness for debriefing and education. Lastly, debriefing was incorporated into biweekly resident mock codes by asking residents to practice using the DEBRIEF after each scenario.

Responses to Likert scale survey questions were dichotomized for analysis (ie, strongly agree and agree were combined into a single category). We used descriptive statistics and $\chi^{2}$ tests to analyze quantitative data. A $P$ value of $<.05$ was considered significant. Statistical process control methodology ${ }^{13}$ was used to track changes in debriefing frequency as reported by real-time RRT-A feedback forms. Random, common cause variation was differentiated from special cause change attributable to our formal debriefing program using standard industry criteria. ${ }^{14}$

The study was approved by the Columbia University Medical Center Institutional Review Board with a waiver of the requirement for informed consent from participants.

\section{Results}

There were 300 RRT-As in the preintervention year and 285 in the postintervention year. Debriefing after RRT-As increased from a mean of $26 \%$ preintervention to a mean of $46 \%$ postintervention $(P<.0001$; FIGURE 1$)$. To test for longevity of effect, we examined the debriefing rate from October to December 2016, a period of time that ranged 3 to 6 


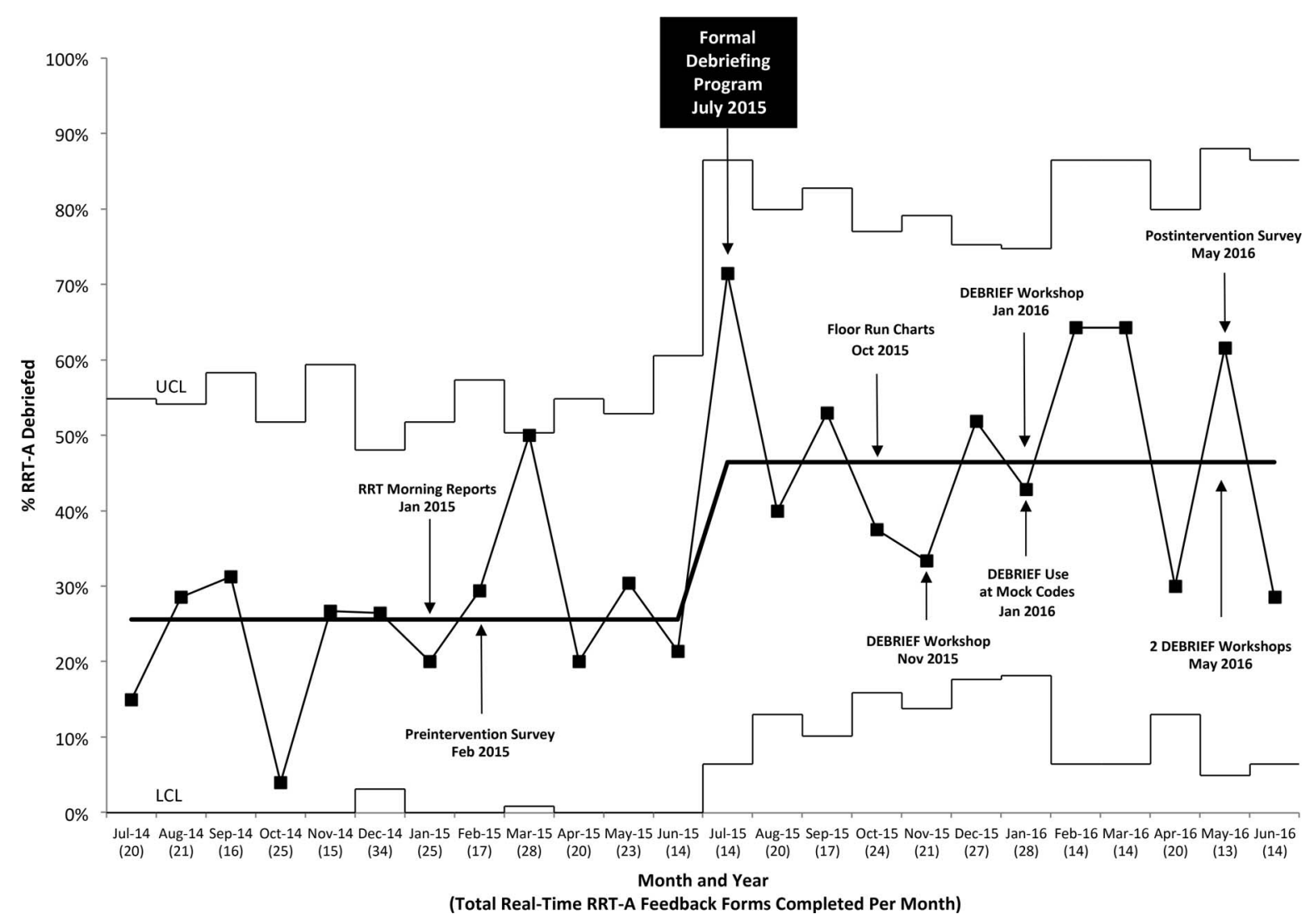

FIGURE 1

P-Control Chart of Rapid Response Team Activation (RRT-A) Debriefing Frequency (July 2014-June 2016)

Note: Abbreviations: UCL, upper control limit; LCL, lower control limit.

months after the study period ended, and found it remained stable at $46 \%$. The real-time RRT-A feedback form response rates were similar preintervention and postintervention at $86 \%$ (257 of $300)$ and $79 \%$ (226 of 285$)$, respectively. Perception of interdisciplinary presence at debriefings (reported on real-time RRT-A feedback forms) showed no change (36\% preintervention to $45 \%$ postintervention, $P=.37)$.

Comparing preintervention versus postintervention periods, physiology was discussed in $65 \%$ (24 of 37) versus $79 \%$ (81 of 102$)$ of debriefings $(P=.12)$, communication in $16 \%$ (6 of 37 ) versus $66 \%$ (67 of $102, P \leq .0001)$, and event anticipation in $59 \%(22$ of 37 ) versus $55 \%$ (56 of $102, P=.78$ ).

According to data collected from the real-time RRT-A feedback forms over the 2-year study period, issues with noise, crowding, and equipment occurred less than $10 \%$ of the time, both preintervention and postintervention. PICU fellow attendance and patient flow remained stable during the study period (FIGURE 2).

A total of 43 of 76 pediatrics residents $(57 \%)$ attended at least 1 DEBRIEF workshop, which was offered on 4 separate dates. There were 4 residents who attended twice, for a total of 47 participants. A total of 45 evaluation forms were completed $(96 \%$ completion rate). After participating in this workshop, 96\% (43 of 45) of participants agreed or strongly agreed that their confidence to lead debriefings after RRT-As had improved, and 98\% (44 of 45) reported a greater willingness to lead multidisciplinary debriefing for future RRT-As.

The online survey response rate was $52 \%$ preintervention (81 respondents/155 invitations) and $39 \%$ postintervention (66 respondents/170 invitations). A total of $47 \%$ (37 of 78 ) of preintervention and $55 \%$ (35 of 64 ) of postintervention respondents reported an experience of more than 10 RRT-As. The perception that debriefings after RRT-As occurred never or rarely decreased from $74 \%$ (60 of 81 ) to $58 \%$ (38 of $66, P=.05$ ). Both preintervention and postintervention, more than $80 \%$ (70 of 78 preintervention and 54 of 65 postintervention) of respondents strongly agreed or agreed there was a benefit to debriefing after RRT-As. After establishing a formal debriefing program, we added new questions to the postintervention survey that targeted 


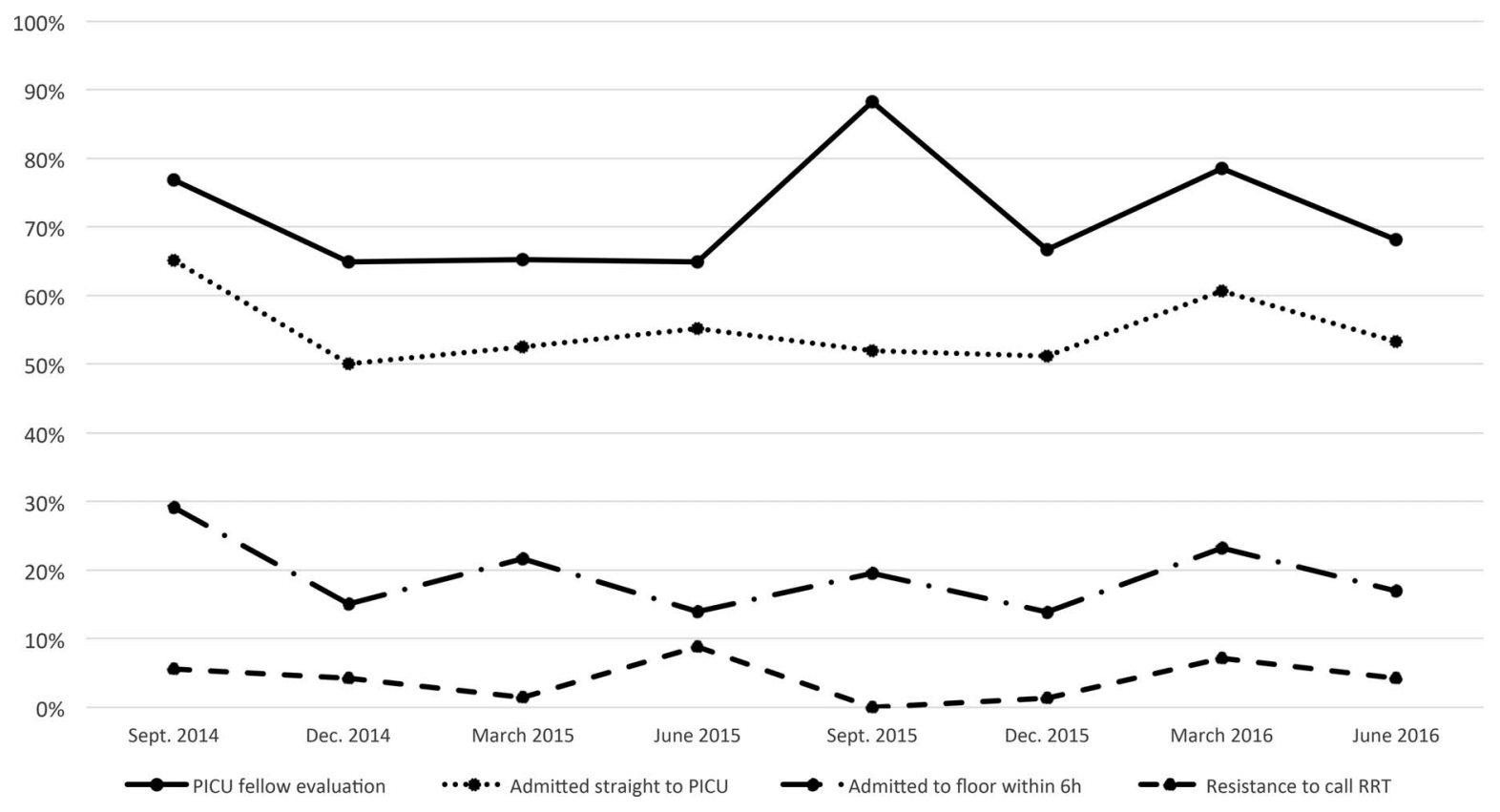

FIGURE 2

Preintervention and Postintervention Data Collected From Real-Time Rapid Response Team Activation (RRT-A) Feedback Forms (September 2014-June 2016)

Note: Abbreviation: PICU, pediatric intensive care unit.

experience with debriefing: $65 \%$ (26 of 40 ) of respondents strongly agreed or agreed that debriefing improved their understanding of the RRT-A process, and $47 \%$ (22 of 47 ) strongly agreed or agreed their comfort level in participating in RRT-As had increased as a result of past RRT-A debriefing sessions. The biggest obstacles to debriefing after RRT-As were reported as "feeling too busy" (47\%, 30 of 64$)$, participants "forgetting" (19\%, 12 of 64$)$, and feeling that "no debriefing was necessary" (16\%, 10 of 64; TABLE). The most frequent RRT-A debriefing leader was the pediatrics resident in both the preintervention and postintervention surveys: $45 \%$ preintervention to $60 \%$ postintervention $(P=.27)$. RRT-A characteristics, including patient floor-PICU flow and PICU fellow attendance at RRT-As, remained stable preintervention and postintervention, suggesting that RRT-A throughput was not affected.

\section{Discussion}

The goal of our study was to use debriefing sessions after RRT-As as learning opportunities. Formal debriefing allowed RRT-A participants to focus on physiology and communication, emphasizing the highest-yield RRT-A learning points. Nearly half of health professionals and trainees indicated that their comfort levels participating in RRT-As had increased as a result of RRT-A debriefing sessions, suggesting that debriefing may be a useful educational tool.

Most health care providers are capable of leading debriefing, given the proper tools. Even novice instructors using standardized scripts are able to facilitate debriefing in a way that improves learning by participants. ${ }^{12}$ This formal RRT-A debriefing program offers health care professionals and trainees the opportunity for additional educational opportunities from RRT-As using experiential learning as a foundation. The Kolb theory of experiential learning defines 4 elements: concrete experience, observation and reflection, formation of abstract concepts, and testing in new situations. ${ }^{15}$ Debriefing after RRT-As is a form of "observation and reflection," with the goal that debriefing will influence participants' synthesis of information. Our hope is that debriefing after RRT-As will lead to the "formation of concepts" that can be applied to the next RRT-A ("new situations").

There are several limitations to our study. First, the preintervention and postintervention surveys were sent to all RRT members, and responses were not stratified by profession. Thus, we are not able to determine resident-specific answers to the questions, and this may overestimate or underestimate the degree to which residents found the intervention educational. Second, our survey response rates were relatively low, limiting internal validity and generalizability. Finally, our $P$ level was not corrected for 
Preintervention and Postintervention Survey Responses ${ }^{a}$

\begin{tabular}{|l|c|c|}
\hline \multicolumn{1}{|c|}{ Survey Item } & $\begin{array}{c}\text { Preintervention, } \mathbf{n} \\
\text { (\% Responses) }\end{array}$ & $\begin{array}{c}\text { Postintervention, } \\
\text { (\% Responses) }\end{array}$ \\
\hline My role in the PICU is: (if reported) & 5 (6) & $5(8)$ \\
\hline PICU fellow & $37(47)$ & $26(42)$ \\
\hline Pediatrics resident & $20(26)$ & $16(26)$ \\
\hline PICU charge nurse & $9(12)$ & $6(10)$ \\
\hline PICU nurse & $7(9)$ & $9(15)$ \\
\hline Respiratory therapist & $60(74)$ & $38(58)$ \\
\hline Debriefing after an RRT activation occurs (never/rarely) & $70(89)$ & $54(83)$ \\
\hline $\begin{array}{l}\text { I (strongly agree/agree) that there is a benefit to having } \\
\text { any form of debriefing after an RRT activation }\end{array}$ & & $26(65)^{\mathrm{b}}$ \\
\hline $\begin{array}{l}\text { I (strongly agree/agree) that having debriefings after RRT activations } \\
\text { helped me understand the RRT activation process }\end{array}$ & & $49(77)$ \\
\hline $\begin{array}{l}\text { It is (very important/important) to have an interdisciplinary team presence } \\
\text { (some combination of MDs, RNs, NPs, RTs) during debriefings after RRT } \\
\text { activations }\end{array}$ & & \\
\hline $\begin{array}{l}\text { In the past year, the biggest obstacle to having formal debriefings after } \\
\text { RRT activations was: felt too busy }\end{array}$ & & \\
\hline $\begin{array}{l}\text { I (strongly agree/agree) that my comfort level in participating in RRT } \\
\text { activations has increased as a result of past RRT debriefing sessions }\end{array}$ & & $30(47)$ \\
\hline
\end{tabular}

Abbreviations: PICU, pediatric intensive care unit; RRT, rapid response team; MD, medical doctor; RN, registered nurse; NP, nurse practitioner; RT, respiratory therapist.

${ }^{a}$ Note: preintervention survey $\mathrm{n}$ range, 78 to 81 ; postintervention survey $\mathrm{n}$ range, 64 to 66 .

${ }^{\mathrm{b}} \mathrm{n}=40$, excluded participants who had not participated in RRT debriefing.

${ }^{c} \mathrm{n}=47$, excluded participants who had not participated in RRT debriefing.

multiple associations, and some of our associations may be spurious.

Future goals include evaluating the optimum frequency for delivering the DEBRIEF workshops to sustain reported debriefing practices (currently offered twice yearly). We also aim to improve interdisciplinary presence at debriefings, and will collect learning points from debriefings to disseminate to all staff members.

\section{Conclusion}

We demonstrated the feasibility and sustainability of a formal debriefing program after pediatric RRT-As. Pediatrics resident-led debriefing huddles after RRTAs led to an increased rate of post-RRT-A debriefing. This multifaceted intervention resulted in residents and RRT members reporting increased occurrence of RRT-A debriefing sessions. The intervention was accomplished with minimal cost, and improvements were sustained 6 months postintervention.

\section{References}

1. Maharaj R, Raffaele I, Wendon J. Rapid response systems: a systematic review and meta-analysis. Crit Care. 2015;19:254.
2. Winters BD, Weaver SJ, Pfoh ER, et al. Rapid-response systems as a patient safety strategy: a systematic review. Ann Intern Med. 2013;158(5, pt 2):417-425.

3. Bhanji F, Mancini ME, Sinz E, et al. Part 16: education, implementation, and teams: 2010 American Heart Association Guidelines for Cardiopulmonary Resuscitation and Emergency Cardiovascular Care. Circulation. 2010;122(18, suppl 3):920-933.

4. Roberts NK, Williams RG, Kim MJ, et al. The briefing, intraoperative teaching, debriefing model for teaching in the operating room. J Am Coll Surg. 2009;208(2):299-303.

5. Cho SJ. Debriefing in pediatrics. Korean J Pediatr. 2015;58(2):47-51.

6. Cheng A, Grant V, Dieckmann P, et al. Faculty development for simulation programs: five issues for the future of debriefing training. Simul Healthc. 2015;10(4):217-222.

7. Garden AL, Le Fevre DM, Waddington HL, et al. Debriefing after simulation-based non-technical skill training in healthcare: a systematic review of effective practice. Anaesth Intensive Care. 2015;43(3):300-308.

8. Paige JT, Arora S, Fernandez G, et al. Debriefing 101: training faculty to promote learning in simulation-based training. Am J Surg. 2015;209(1):126-131.

9. Salas E, Klein C, King H, et al. Debriefing medical teams: 12 evidence-based best practices and tips. $J t$ Comm J Qual Patient Saf. 2008;34(9):518-527. 
10. Balmer DF, Quiah S, DiPace J, et al. Learning across the explicit, implicit, and extra-curricula: an exploratory study of the relative proportions of residents' perceived learning in clinical areas at three pediatric residency programs. Acad Med. 2015;90(11):1547-1552.

11. Zebuhr C, Sutton RM, Morrison W, et al. Evaluation of quantitative debriefing after pediatric cardiac arrest. Resuscitation. 2012;83(9):1124-1128.

12. Cheng A, Hunt EA, Donoghue A, et al. Examining pediatric resuscitation education using simulation and scripted debriefing: a multicenter randomized trial. JAMA Pediatr. 2013;167(6):528-536.

13. Guthrie B, Love T, Fahey T, et al. Control, compare and communicate: designing control charts to summarise efficiently data from multiple quality indicators. Qual Saf Health Care. 2005;14(6):450-454.

14. Amin SG. Control charts 101: a guide to health care applications. Qual Manag Health Care. 2001;9(3):1-27.

15. Kolb DA. Experiential Learning: Experience as the Source of Learning and Development. Englewood Cliffs, NJ: Prentice-Hall; 1984.

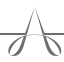

Linda Aponte-Patel, MD, is Assistant Professor, Division of Pediatric Critical Care, and Associate Director, Pediatric Residency Program, Department of Pediatrics, NewYork-Presbyterian Morgan Stanley Children's Hospital, Columbia University Medica Center; Arash Salavitabar, MD, is a Fellow, Division of Pediatric Cardiology, Department of Pediatrics, NewYork-Presbyterian Morgan Stanley Children's Hospital, Columbia University Medical Center; Pamela Fazzio, MD, is a Fellow, Division of Emergency
Medicine, The Children's Hospital of Philadelphia; Andrew S. Geneslaw, MD, is a Fellow, Division of Pediatric Critical Care, Department of Pediatrics, NewYork-Presbyterian Morgan Stanley Children's Hospital, Columbia University Medical Center; Pamela Good, MD, is a Fellow, Division of Neonatology, Department of Pediatrics, NewYork-Presbyterian Morgan Stanley Children's Hospital, Columbia University Medical Center; and Anita I. Sen, MD, is Assistant Professor and Director of Quality, Division of Pediatric Critical Care, Department of Pediatrics, NewYorkPresbyterian Morgan Stanley Children's Hospital, Columbia University Medical Center.

Funding: The authors report no external funding source for this study.

Conflict of interest: The authors declare they have no competing interests.

The study results were presented as a poster at the Pediatric Educational Excellence across the Continuum Meeting, Atlanta, Georgia, September 18-19, 2015, and as a poster at the Pediatric Academic Societies Meeting, San Francisco, California, May 6-9, 2017. The results were also published as abstracts: Aponte-Patel L, Salavitabar A, Fazzio P, et al. Implementation of a formal debriefing program after pediatric rapid response team activations. Crit Care Med. 2016;44(12):389; and Aponte-Patel L, Salavitabar A, Fazzio P, et al. The value of debriefing after rapid response team activations at a tertiary-care children's hospital. Crit Care Med. 2015;43(12 suppl 1):53.

The authors would like to thank Dr Pooja Desai-England, Dr Roselle Vittorino, Dr John Babineau, all of our pediatrics residents, and pediatric intensive care unit fellows, nurses, and respiratory therapists for their phenomenal patient care.

Corresponding author: Linda Aponte-Patel, MD, NewYorkPresbyterian/Columbia University Medical Center, CHN 10-24, 3959 Broadway, New York, NY 10032, 212.305.8458, la2423@cumc.columbia.edu

Received July 19, 2017; revisions received November 7, 2017, and December 21, 2017; accepted January 8, 2018. 